\title{
An Update on Ocular Surface Epithelial Stem Cells: Cornea and Conjunctiva
}

\author{
Tiago Ramos, ${ }^{1}$ Deborah Scott, ${ }^{1}$ and Sajjad Ahmad ${ }^{1,2}$ \\ ${ }^{1}$ Department of Eye and Vision Science, Institute of Ageing and Chronic Disease, University of Liverpool, Liverpool L69 3GA, UK \\ ${ }^{2}$ St. Paul's Eye Unit, Royal Liverpool University Hospital, Liverpool, UK \\ Correspondence should be addressed to Tiago Ramos; t.ramos@liverpool.ac.uk
}

Received 26 February 2015; Revised 21 May 2015; Accepted 25 May 2015

Academic Editor: Toru Hosoda

Copyright (c) 2015 Tiago Ramos et al. This is an open access article distributed under the Creative Commons Attribution License, which permits unrestricted use, distribution, and reproduction in any medium, provided the original work is properly cited.

\begin{abstract}
The human ocular surface (front surface of the eye) is formed by two different types of epithelia: the corneal epithelium centrally and the conjunctival epithelium that surrounds this. These two epithelia are maintained by different stem cell populations (limbal stem cells for the corneal epithelium and the conjunctival epithelial stem cells). In this review, we provide an update on our understanding of these epithelia and their stem cells systems, including embryology, new markers, and controversy around the location of these stem cells. We also provide an update on the translation of this understanding into clinical applications for the treatment of debilitating ocular surface diseases.
\end{abstract}

\section{Introduction}

The front of the human eye is formed by the clear cornea centrally and the white sclera peripherally (Figure 1). The cornea and sclera are covered by the corneal and conjunctival epithelia, respectively. These epithelia are nonkeratinized and stratified structures. The corneal epithelium is 5-7 cell layers thick whereas the conjunctival epithelium is $3-5$ cell layers thick. These two epithelial structures are bathed in a tear film, and together these form the front surface of the eye (the ocular surface) $[1,2]$. An important function of the ocular surface (cornea, conjunctiva, and the overlying tear film) is to protect the eye from injury, infection, and desiccation [2]. The cornea is a clear structure that is composed of five main layers and its main function is to transmit and focus light into the eye. The superficial layers of the cornea (the stratified epithelium, Bowman's layer, and the superficial stroma) are continuous with the conjunctiva. The conjunctiva is a thin loose transparent mucous layer covering the anterior surface of the globe and the posterior surface of the eyelids [2]. The conjunctiva is divided into three regions: bulbar (covering the surface of the eye), palpebral (lining the undersurface of the eyelids), and the forniceal region in between. The conjunctival epithelium contains superficial scattered goblet cells that produce important mucins for the tear film. Physically separating the corneal epithelium and the conjunctival epithelium is a narrow band of limbal epithelium that encircles the cornea. The limbal epithelium acts as a barrier between the clear avascular cornea centrally and the opaque vascularized conjunctiva peripherally. The limbal epithelium also contains the stem cells (SCs) that renew the corneal epithelium, the limbal SCs (LSCs) [3, 4].

Both corneal and conjunctival epithelia are susceptible to a wide range of diseases from injuries such as chemical burns to inflammatory diseases such as mucous membrane pemphigoid and Stevens-Johnson syndrome. These can result in significant visual impairment and ocular surface pain. Different treatment modalities have already been presented as a therapy for limbal SC deficiency (LSCD) with significant clinical improvements $[5,6]$. However, the approaches for developing conjunctival epithelial constructs for scarring conjunctival diseases are still very limited [7].

In the present review we provide a concise update on ocular surface epithelial SCs: their location, distribution, and the markers used to identify them. The clinical applications of corneal and conjunctival epithelial and their SCs will also be discussed. 


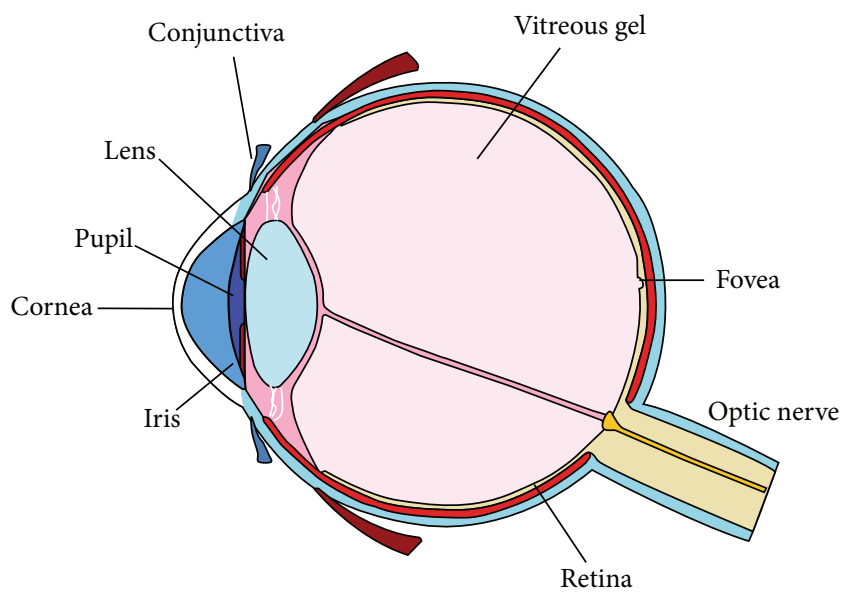

FIGURE 1: Cross-sectional illustrative view of the adult human eye.

\section{Embryological Origins of the Ocular Surface Epithelia}

In order for us to understand ocular surface epithelial SC biology, it is important to understand the embryological origin of the corneal epithelium and the conjunctival epithelium. It has been shown that these two cell lineages arise simultaneously from Pax $6^{+}$ectodermal cells that remain on the embryonic ectodermal surface of the developing eye once the lens vesicle has formed $[8,9]$. The PAX6 gene encodes a transcription factor critical for normal embryonic development. The PAX6 protein is expressed in the developing eye, multiple brain regions, olfactory bulb, neural tube, gut, and pancreas [10]. In humans, insufficient PAX6 protein expression results in severe congenital defects of the eye [11]. It is therefore considered the master gene for oculogenesis.

The developing human corneal epithelium is first apparent at 6 weeks after ovulation [12]. The primitive corneal epithelium is initially composed of two cell layers (as compared to the five to seven layers in the adult). This primitive epithelium is also responsible for forming a prominent primary acellular corneal stroma and Bowman's layer [13]. Sometime between 8 weeks of gestation (when the eyelids fuse together) and 26 weeks of gestation (when eyelids open), the corneal epithelium stratifies to four to five cell layers thick. Adhesion complexes only become detectable by 19 weeks of gestation. The further development in utero leads to an increase in the number of hemidesmosomes, an increase in the fibril penetration into Bowman's layer, and an increase in Bowman's layer thickness. Maturation of the corneal epithelium is therefore related to eyelid development [12].

Despite their closeness, the corneal ECs and the conjunctival ECs belong to distinct lineages $[14,15]$ arising from different cell populations [16]. In vivo studies in rabbit have shown that limbal and corneal EC derived cysts contained only stratified squamous-type ECs. In contrast, conjunctival EC derived cysts contained stratified columnar-type ECs interspersed with periodic acid-Schiff (PAS) staining cells (PAS is a mucin stain) with a goblet-like structure (the goblet cells) [16]. This supports the hypothesis that corneal and limbal ECs originate along a different embryonic lineage to conjunctival ECs, and that the goblet cells originate from the conjunctival compartment.

\section{Conjunctival Epithelium and Its Stem Cells}

3.1. Location of Conjunctival Stem Cells (CjSCs). Whilst it is commonly regarded that the SCs for the corneal epithelium (the LSCs) are located in the limbal epithelium, the location of SCs for the conjunctival epithelium is more controversial. The following regions of the conjunctiva have been proposed as possible sites for the SCs: the fornix region [17], the limbus [18], bulbar conjunctiva [19], palpebral conjunctiva [20], and at the mucocutaneous junction on the eyelid margin [21]. Bromodeoxyuridine label retention (a property of quiescent SCs) in GFP-labelled mice suggests that epithelial SCs in the conjunctiva are uniformly distributed throughout the whole surface [22]. Another important property of epithelial SCs is their ability to initiate clonal growth in vitro and yield colonies consisting of small cells that have a long survival time (called holoclones). By analyzing epithelial colonies cultured from different regions of the human conjunctiva, Pellegrini et al. were the first to suggest that conjunctival epithelial SCs are located uniformly in the bulbar conjunctiva and the fornices [19]. More recently, Stewart et al. using both clonogenic ability and expression of putative SCs markers (ABCG2 and p63) showed that the medial canthal and the inferior forniceal areas are the preferred area for the human CjSCs. They suggested that those areas provide greater physical protection but more importantly are especially rich in goblet cells, intraepithelial mucous crypts, blood vessels, melanocytes, and immune cells, features shared with other SC niches [23]. In other studies, where the slow cell-cycling and the great proliferative potential were analyzed it has been shown that the forniceal region of the rabbit conjunctiva contains the largest proportions of cells with high proliferative potential and a higher percentage of slow cycling cells (14\% of ECs) than the bulbar (5\%) and palpebral conjunctiva $(1 \%)[15,17]$.

Although there are no specific markers for CjSCs, ABCG2 positive cells have been found in clusters of human bulbar conjunctival epithelial basal cells and these cells display many features that are consistent with the epithelial SC phenotype, slow cycling, clonogenic capacity, and resistance to phorbolinduced differentiation [24]. Other immunohistological studies also suggest the presence of p63 and ABCG2 positive cells in the bulbar conjunctival epithelium [25]. Clinical observations also indicate that the CjSCs are located in the fornix and/or in the bulbar conjunctiva [26].

Despite the different observations, resulting from differing techniques and species used, all seem to point in the higher amount of conjunctival SCs in the forniceal area. The fornix may provide greater physical protection, intraepithelial mucous crypts, vasculature, and immune cells, features shared with other SCs niches [23].

3.2. Markers for Conjunctival Epithelium and Its SCs. The identification of a marker that is expressed in the conjunctival epithelium but not in the corneal epithelium has been a growing need. Because of their different patterns of expression, 
TABLE 1: A table showing the distribution of different keratins across the ocular surface epithelia (corneal, limbal, and conjunctival).

\begin{tabular}{lccc}
\hline Cytokeratin & Cornea & Limbus & Conjunctiva \\
\hline CK1 2,10 & + & + & ++ \\
CK3 & $\begin{array}{c}\text { Periphery: basal and } \\
\text { intermediate cells }+++ \\
\text { Centre: all cells }++\end{array}$ & +++ & ++ \\
\hline CK5 & +++ & +++ & Basal cells,+++ intermediate cells ++ \\
\hline CK14-18 & +++ & Basal and superficial cells +++ & +++ \\
\hline CK1-8 and K5, 10, 11 & +++ & +++ & +++ \\
\hline CK8, 18, 19 & +++ & Superficial and intermediate cells ++ & +++ \\
\hline
\end{tabular}

cytokeratins (CKs) have been widely used to distinguish the different ECs of the ocular surface [27] (Table 1). CKs are intermediate filament-forming proteins responsible for the structural integrity and function of ECs [28]. Different CKs have been suggested as specific markers for conjunctival ECs, amongst those are CK4, CK13, CK19, and CK15.

CK4: In mouse conjunctiva, CK4 is expressed in all the epithelial layers, but the expression in the basal layers seems to be weaker and more focal. However, CK4 is also expressed in mouse and rabbit superficial layers of the corneal epithelium in vivo as well $[29,30]$.

CK13 and CK19: CK13 and CK19 are the most accepted markers for conjunctival ECs with confounding results arising from different investigations. Ramirez-Miranda et al. have shown that CK13 and CK19 expression is significantly increased in the human conjunctiva in comparison to the human cornea. They have shown that CK13 is expressed only in the suprabasal limbal epithelium and in all layers of the conjunctival epithelia and it is completely absent in the cornea. On the other hand, CK19 is present at substantial levels in the peripheral corneal epithelium and in all layers of the limbus and conjunctiva epithelium [28, 31,32]. They showed that none of the CK12-positive cells expressed CK13 in the central cornea and the limbal ECs expressed either CK12 or CK13 (CK12 is known to be a specific marker for corneal EC; see Section 4.2). On other hand, CK12 and CK19 positive cells were colocated throughout the limbus and peripheral cornea suggesting a higher specificity of CK13 for conjunctival ECs [28].

CK15: Other investigators have also studied the expression pattern of CK15, a minor cytoskeletal component of stratified tissue proposed to be a marker for progenitor cells [33]. All studies revealed that CK15 is expressed in the basal layers of the limbal and conjunctival epithelia but it is absent in the corneal epithelium $[34,35]$. Other in vitro studies have also shown the expression of CK15 by conjunctival epithelial progenitor cells but they suggest that more differentiated cells may also express this marker [36].

Other markers for conjunctival epithelial cells are the mucins. Epithelial MUCs are a heterogeneous group of large glycosylated proteins, which form the viscous, gel-like mucous layer of the tear film. Both MUC1 and MUC5AC have been postulated as being markers for conjunctival ECs and/or goblet cells.

MUC1: The exclusivity of MUC1 to conjunctival ECs is debated with some proposing it as a conjunctival epithelial specific marker [37] and others suggesting expression throughout the entire ocular surface in health $[38,39]$.

MUC5AC: MUC5AC, on the other hand, has long been postulated as being specific to the conjunctival goblet cells [40]. Although it is not detected in the ECs of the conjunctiva, it is the best surrogate marker for the presence of conjunctival epithelium by identifying the goblet cells that are absent in the corneal epithelium in health. Another method for identifying goblet cells is by PAS staining of MUCs. This is indeed used clinically in the investigation of corneal conjunctivalisation by corneal impression cytology [40].

There are few studies looking at identifying markers for CjSCs. The most commons are ATP-binding cassette subfamily G member 2 (ABCG2) and the transcription factor p63. ABCG2 is a cell surface transmembrane transporter that is present in many adult SCs, including LSCs. Conceptually, it may form a component of the molecular mechanisms by which long-lived SCs reduce the potential for genomic damage over their extended lives, and their expression has been correlated with SC activity [41]. In human conjunctiva its expression has been found in clusters of basal [24], medial canthal and the inferior forniceal areas conjunctival ECs [23]. Those ABCG2 positive cells display many features that are consistent with the epithelial SC phenotype, slow cycling, clonogenic capacity, and resistance to phorbol-induced differentiation. p63 is a transcription factor that is known to be expressed by LSCs and early transient amplified cells [42-44]. Stewart et al. using cytochemistry analysis showed a preferred location of this transcription factor in the inferior forniceal and medial canthal. The cells in those areas exhibited the higher clonogenic capacities which suggest a phenotype that is consistent with SCs [23].

\section{Limbal SCs}

4.1. Location of Limbal SCs. In corneal epithelial homeostasis the epithelium is constantly renewed to replace desquamating 


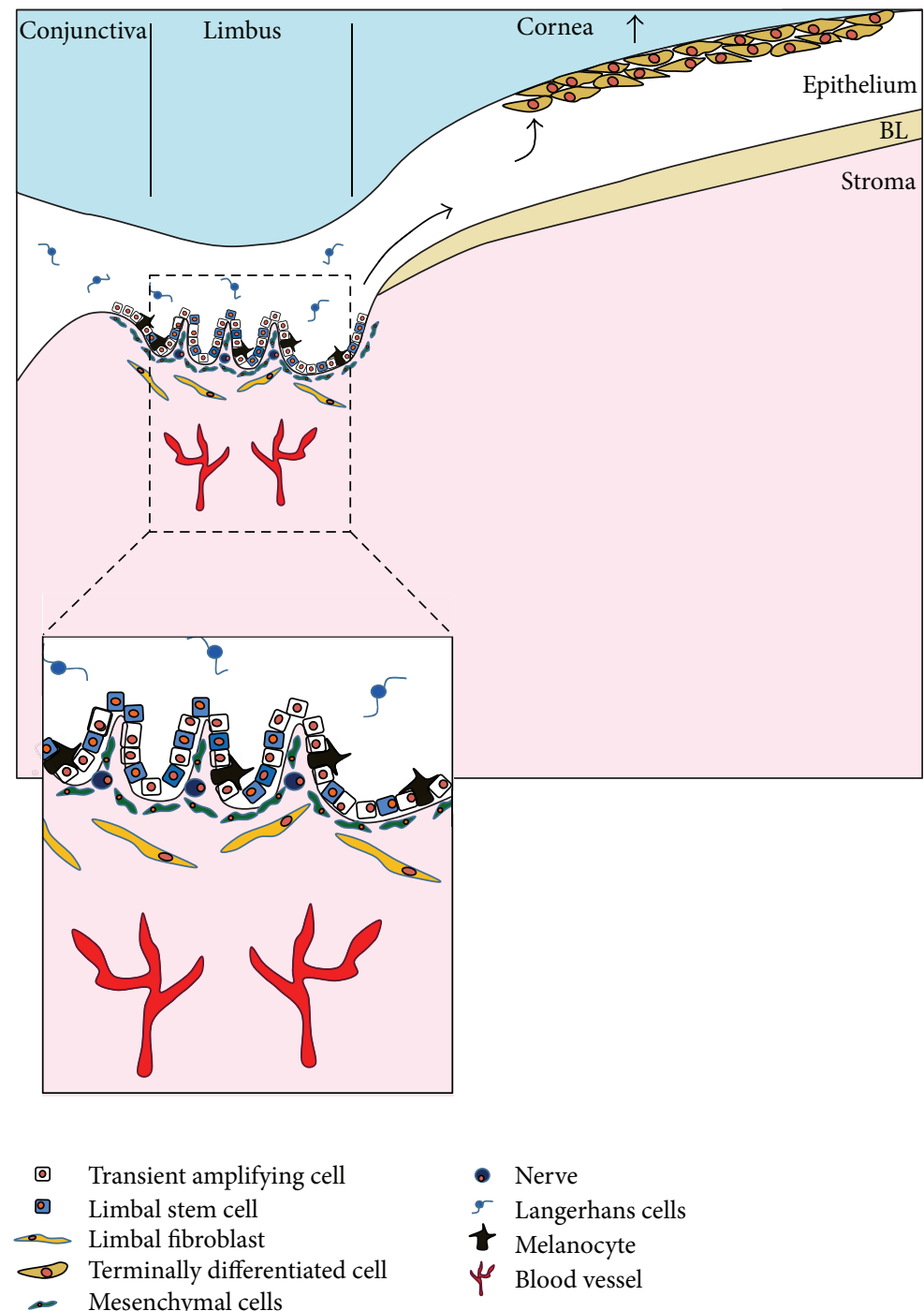

FIGURE 2: Limbal SCs for the corneal epithelium reside in the basal layer of the limbal epithelium. Transient amplifying cells divide and migrate towards the central cornea to replace the terminal corneal ECs that get shed from the corneal surface. The stroma of the limbal SC niche is populated by fibroblasts and nourished by blood vessels (BL: Bowman's layer).

cells that are shed from the corneal surface (Figure 2). This homeostasis is outlined in the XYZ hypothesis of corneal epithelial maintenance [45]. In brief, this describes the proliferation and motility of basal ECs at the periphery of the cornea centripetally along the basement membrane towards the center of the cornea (component $\mathrm{X}$ ) and then the movement of cells from the basal layers to the superficial corneal epithelial layers (component Y) to replace the ECs that are shed from the corneal surface (component $Z$ ).

It is widely accepted that the corneal epithelial equilibrium described above is maintained by the self-renewing undifferentiated SCs located at the limbus. A consistent body of evidence demonstrates the limbal location of corneal epithelial SCs with the original studies dating back to the late 1980s [46]. In brief, the evidence for this includes the following: the presence of basal limbal ECs with a high nucleus to cytoplasm ratio and putative protein expression consistent with SCs $[47,48]$, using DNA-labelling and cellcycling studies, the presence of limbal ECs in a quiescent state but with a high proliferative response in injury; and clinical observations on limbal and corneal epithelial wound healing in humans.

Recent studies in animal models however suggest that there is also a reservoir of SCs within the corneal epithelium itself in addition to the LSCs. Recent nonhuman studies have shown corneal epithelial maintenance without limbal input and survival and self-maintenance of SCs outside the limbal SC niche [49]. Successful corneal epithelial regeneration by sequential corneal epithelial transplantation in a murine model was first proposed as evidence for this [50]. Recently, again in a murine model, lineage-tracing experiments have shown that although the limbus is the prime source for corneal epithelial maintenance, there is also a reservoir of clonogenic cells within the corneal epithelium itself [51]. In 
addition to these studies, 48-week follow-up rabbit studies have shown that although normal corneal epithelium cannot initially be maintained following the removal of limbal epithelium, there is evidence of corneal epithelial normalization at 48 weeks [52]. Bringing together all the evidence available, although corneal epithelial maintenance relies upon SCs within the limbus, there is some evidence in animal models that there may also be a pool of progenitor cells within the corneal epithelium itself when homeostasis is compromised [53]. Further research is required particularly in human studies to provide strength and consistency to this theory.

4.2. Markers for Limbal SCs. Despite decades of research, no definitive marker for LSCs has been identified. Comprehensive reviews of putative suggested LSC markers have been published elsewhere $[54,55]$. In brief, putative positive markers include the transcription factor $\triangle \mathrm{Np} 63 \alpha$ and the ABCG2; whilst negative markers include $\mathrm{CK} 3$ and $\mathrm{CK} 12$, the structural proteins found in the corneal epithelium. More recently, murine and human studies have shown that cells expressing the ATP-binding cassette, subfamily B, member 5 (ABCB5) are required for corneal epithelial homeostasis and repair [56]. They showed that ABCB5 expressing cells were localized to the limbus and coexpressed $\Delta \mathrm{Np} 63 \alpha$ but not CK12.

\section{The Clinical Applications of Corneal and Conjunctival Epithelia and Their SCs}

Diseases of the corneal and conjunctival epithelia result in debilitating and blinding eye diseases that are often chronically painful. These diseases result from injury such as burns, inflammatory and immune mediated diseases (such as mucous membrane pemphigoid), and iatrogenic causes (such as radiotherapy and chemotherapy). Our understanding of corneal and conjunctival epithelial biology and increasing knowledge of their SCs is critical to the clinical management of these diseases.

Limbal SC failure results in an abnormally regenerating corneal epithelium that then gets replaced by a more opaque conjunctival epithelium and blood vessels. This results in loss of vision. Transplanting healthy limbal tissue or cultured limbal epithelium containing limbal SCs has been shown in numerous studies to result in normalization of the corneal epithelium [5, 6]. Using DNA fingerprinting methods in allogeneic transplants, it has been shown that often the donor cells do not survive on the corneal surface despite the corneal epithelium regenerating normally [57]. This then begs the question as to how the transplanted limbal epithelium and limbal SCs contribute to normalization of the host corneal epithelium. Many now believe that paracrine influences from the transplanted ECs may result in host SC recovery or certainly contribute to it. It is also interesting to note that the success rate for cultured limbal epithelial transplants is approximately equal for both allogeneic and autologous transplants (about 75\% success) [58]. This may suggest that transplant failure is less likely to result from rejection and that other factors may well be at play (such as the number of limbal SCs in the transplanted tissue).

As outlined above, although improved knowledge of limbal SC biology has resulted in significant advances in clinical benefit, it has been slower with regard to the conjunctival epithelium. However, our understanding of conjunctival epithelial SCs and their culture has improved. Many groups are now working on developing conjunctival epithelial constructs for scarring conjunctival diseases with still very limited results [7]. Regarding conjunctival epithelium, as our understanding of conjunctival epithelial SCs improves, clinical applications of this biology will bring considerable benefit. There are already several groups working on developing conjunctival epithelial constructs for scarring conjunctival diseases. It has even been suggested that cultivated conjunctival epithelium may be used as a treatment for limbal SC and corneal epithelial failure [7].

\section{Conclusion}

Our knowledge of limbal SC and conjunctival epithelial SC biology has progressed considerably in the last two decades with significant clinical advances being made in limbal SC transplantation. There however remain critical areas for further work. These include more understanding of the cellular biology of these SCs and in the process the identification of further markers. This will enable the development of nonsurgical approaches (drug and biological agents) to the management of ocular surface epithelial diseases. In addition, the location of these SCs is important to understand fully. There are emerging concepts that are beginning to challenge our conventional understanding of this.

\section{Abbreviations \\ SC: $\quad$ Stem cell \\ EC: Epithelial cell \\ LSC: Limbal stem cells \\ CjSC: Conjunctival stem cell \\ CK: Cytokeratin \\ MUC: Mucin.}

\section{Conflict of Interests}

The authors declare that there is no conflict of interests regarding the publication of this paper.

\section{Acknowledgments}

This work was supported by a grant from Crossley Barnes Bequest Fund and Fight for Sight.

\section{References}

[1] S. Schrader, M. Notara, M. Beaconsfield, S. J. Tuft, J. T. Daniels, and G. Geerling, "Tissue engineering for conjunctival reconstruction: established methods and future outlooks," Current Eye Research, vol. 34, no. 11, pp. 913-924, 2009. 
[2] N. Di Girolamo, "Stem cells of the human cornea" British Medical Bulletin, vol. 100, no. 1, pp. 191-207, 2011.

[3] S. Ahmad, F. Figueiredo, and M. Lako, "Corneal epithelial stem cells: characterization, culture and transplantation," Regenerative Medicine, vol. 1, no. 1, pp. 29-44, 2006.

[4] S. Kolli, S. Ahmad, H. S. Mudhar, A. Meeny, M. Lako, and F. C. Figueiredo, "Successful application of ex vivo expanded human autologous oral mucosal epithelium for the treatment of total bilateral limbal stem cell deficiency," Stem Cells, vol. 32, no. 8, pp. 2135-2146, 2014.

[5] K. R. Kenyon and S. C. G. Tseng, "Limbal autograft transplantation for ocular surface disorders," Ophthalmology, vol. 96, no. 5, pp. 709-723, 1989.

[6] P. Rama, S. Bonini, A. Lambiase et al., "Autologous fibrincultured limbal stem cells permanently restore the corneal surface of patients with total limbal stem cell deficiency," Transplantation, vol. 72, no. 9, pp. 1478-1485, 2001.

[7] L. P. K. Ang, H. Tanioka, S. Kawasaki et al., "Cultivated human conjunctival epithelial transplantation for total limbal stem cell deficiency," Investigative Ophthalmology and Visual Science, vol. 51, no. 2, pp. 758-764, 2010.

[8] J. A. Davis and R. R. Reed, "Role of Olf- 1 and Pax-6 transcription factors in neurodevelopment," The Journal of Neuroscience, vol. 16, no. 16, pp. 5082-5094, 1996.

[9] B. M. Koroma, J.-M. Yang, and O. H. Sundin, "The Pax6 homeobox gene is expressed throughout the corneal and conjunctival epithelia," Investigative Ophthalmology \& Visual Science, vol. 38, no. 1, pp. 108-120, 1997.

[10] H. J. Lee and K. A. Colby, "A review of the clinical and genetic aspects of aniridia," Seminars in Ophthalmology, vol. 28, no. 5-6, pp. 306-312, 2013.

[11] J. Ou, C. Lowes, and J. M. Collinson, "Cytoskeletal and cell adhesion defects in wounded and Pax6 $6^{+/-}$corneal epithelia," Investigative Ophthalmology and Visual Science, vol. 51, no. 3, pp. 1415-1423, 2010.

[12] D. Sevel, R. Isaacs, and A. P. Ferry, "A re-evaluation of corneal development," Transactions of the American Ophthalmological Society, vol. 86, pp. 178-207, 1988.

[13] D. G. Dawson, M. A. Watsky, D. H. Geroski, and H. F. Edelhauser, Duane's Foundations of Clinical Ophthalmology, vol. 2, 2007.

[14] F. E. Kruse, J. J. Y. Chen, R. J. F. Tsai, and S. C. G. Tseng, "Conjunctival transdifferentiation is due to the incomplete removal of limbal basal epithelium," Investigative Ophthalmology \& Visual Science, vol. 31, no. 9, pp. 1903-1913, 1990.

[15] Z.-G. Wei, R.-L. Wu, R. M. Lavker, and T.-T. Sun, "In vitro growth and differentiation of rabbit bulbar, fornix, and palpebral conjunctival epithelia: implications on conjunctival epithelial transdifferentiation and stem cells," Investigative Ophthalmology \& Visual Science, vol. 34, no. 5, pp. 1814-1828, 1993.

[16] Z.-G. Wei, T.-T. Sun, and R. M. Lavker, "Rabbit conjunctival and corneal epithelial cells belong to two separate lineages," Investigative Ophthalmology and Visual Science, vol. 37, no. 4, pp. 523-533, 1996.

[17] Z.-G. Wei, G. Cotsarelis, T.-T. Sun, and R. M. Lavker, "Labelretaining cells are preferentially located in fornical epithelium: implications on conjunctival epithelial homeostasis," Investigative Ophthalmology and Visual Science, vol. 36, no. 1, pp. 236246, 1995.

[18] J. Peer, G. Zajicek, H. Greifner, and M. Kogan, "Streaming conjunctiva," Anatomical Record, vol. 245, no. 1, pp. 36-40, 1996.
[19] G. Pellegrini, O. Golisano, P. Paterna et al., "Location and clonal analysis of stem cells and their differentiated progeny in the human ocular surface," Journal of Cell Biology, vol. 145, no. 4, pp. 769-782, 1999.

[20] W. Chen, M. Ishikawa, K. Yamaki, and S. Sakuragi, "Wistar rat palpebral conjunctiva contains more slow-cycling stem cells that have larger proliferative capacity: implication for conjunctival epithelial homeostasis," Japanese Journal of Ophthalmology, vol. 47, no. 2, pp. 119-128, 2003.

[21] J. D. Wirtschafter, J. M. Ketcham, R. J. Weinstock, T. Tabesh, and L. K. McLoon, "Mucocutaneous junction as the major source of replacement palpebral conjunctival epithelial cells," Investigative Ophthalmology and Visual Science, vol. 40, no. 13, pp. 3138-3146, 1999.

[22] T. Nagasaki and J. Zhao, "Uniform distribution of epithelial stem cells in the bulbar conjunctiva," Investigative Ophthalmology and Visual Science, vol. 46, no. 1, pp. 126-132, 2005.

[23] R. M. Stewart, C. M. Sheridan, P. S. Hiscott, G. Czanner, and S. B. Kaye, "Human conjunctival stem cells are predominantly located in the medial canthal and inferior forniceal areas," Investigative Ophthalmology \& Visual Science, vol. 56, no. 3, pp. 2021-2030, 2015.

[24] M. T. Budak, O. S. Alpdogan, M. Zhou, R. M. Lavker, M. A. M. Akinci, and J. M. Wolosin, "Ocular surface epithelia contain ABCG2-dependent side population cells exhibiting features associated with stem cells," Journal of Cell Science, vol. 118, part 8, pp. 1715-1724, 2005.

[25] S. G. Vascotto and M. Griffith, "Localization of candidate stem and progenitor cell markers within the human cornea, limbus, and bulbar conjunctiva in vivo and in cell culture," Anatomical Record Part A: Discoveries in Molecular, Cellular, and Evolutionary Biology, vol. 288, no. 8, pp. 921-931, 2006.

[26] K. Tsubota, S. Shimmura, N. Shinozaki, E. J. Holland, and J. Shimazaki, "Clinical application of living-related conjunctivallimbal allograft," The American Journal of Ophthalmology, vol. 133, no. 1, pp. 134-135, 2002.

[27] R. P. Revoltella, S. Papini, A. Rosellini, and M. Michelini, "Epithelial stem cells of the eye surface," Cell Proliferation, vol. 40, no. 4, pp. 445-461, 2007.

[28] A. Ramirez-Miranda, M. N. Nakatsu, S. Zarei-Ghanavati, C. V. Nguyen, and S. X. Deng, "Keratin 13 is a more specific marker of conjunctival epithelium than keratin 19," Molecular Vision, vol. 17, pp. 1652-1661, 2011.

[29] M. A. Kurpakus, M. T. Maniaci, and M. Esco, "Expression of keratins K12, K4 and K14 during development of ocular surface epithelium," Current Eye Research, vol. 13, no. 11, pp. 805-814, 1994.

[30] D. Meller and S. C. G. Tseng, "Conjunctival epithelial cell differentiation on amniotic membrane," Investigative Ophthalmology \& Visual Science, vol. 40, no. 5, pp. 878-886, 1999.

[31] B. C. R. Marsh, M. Massaro-Giordano, C. M. Marshall, R. M. Lavker, and P. J. Jensen, "Initiation and characterization of keratinocyte cultures from biopsies of normal human conjunctiva," Experimental Eye Research, vol. 74, no. 1, pp. 61-69, 2002.

[32] R. Moll, W. W. Franke, D. L. Schiller, B. Geiger, and R. Krepler, "The catalog of human cytokeratins: patterns of expression in normal epithelia, tumors and cultured cells," Cell, vol. 31, no. 1, pp. 11-24, 1982.

[33] S. Lyle, M. Christofidou-Solomidou, Y. Liu, D. E. Elder, S. Albelda, and G. Cotsarelis, "The C8/144B monoclonal antibody recognizes cytokeratin 15 and defines the location of human hair 
follicle stem cells," Journal of Cell Science, vol. 111, part 21, pp. 3179-3188, 1998.

[34] S. Merjava, A. Neuwirth, M. Tanzerova, and K. Jirsova, "The spectrum of cytokeratins expressed in the adult human cornea, limbus and perilimbal conjunctiva," Histology and Histopathology, vol. 26, no. 3, pp. 323-331, 2011.

[35] S. Yoshida, S. Shimmura, T. Kawakita et al., "Cytokeratin 15 can be used to identify the limbal phenotype in normal and diseased ocular surfaces," Investigative Ophthalmology and Visual Science, vol. 47, no. 11, pp. 4780-4786, 2006.

[36] S. Schrader, M. Notara, S. J. Tuft, M. Beaconsfield, G. Geerling, and J. T. Daniels, "Simulation of an in vitro niche environment that preserves conjunctival progenitor cells," Regenerative Medicine, vol. 5, no. 6, pp. 877-889, 2010.

[37] V. Barbaro, S. Ferrari, A. Fasolo et al., "Evaluation of ocular surface disorders: a new diagnostic tool based on impression cytology and confocal laser scanning microscopy," British Journal of Ophthalmology, vol. 94, no. 7, pp. 926-932, 2010.

[38] T. Inatomi, S. Spurr-Michaud, A. S. Tisdale, Q. Zhan, S. T. Feldman, and I. K. Gipson, "Expression of secretory mucin genes by human conjunctival epithelia," Investigative Ophthalmology and Visual Science, vol. 37, no. 8, pp. 1684-1692, 1996.

[39] I. K. Gipson, S. Spurr-Michaud, P. Argüeso, A. Tisdale, T. F. $\mathrm{Ng}$, and C. L. Russo, "Mucin gene expression in immortalized human corneal-limbal and conjunctival epithelial cell lines," Investigative Ophthalmology and Visual Science, vol. 44, no. 6 , pp. 2496-2506, 2003.

[40] I. Garcia, J. Etxebarria, A. Boto-De-Los-Bueis et al., "Comparative study of limbal stem cell deficiency diagnosis methods: detection of MUC5AC mRNA and goblet cells in corneal epithelium," Ophthalmology, vol. 119, no. 5, pp. 923-929, 2012.

[41] K. D. Bunting, "ABC transporters as phenotypic markers and functional regulators of stem cells," Stem Cells, vol. 20, no. 1, pp. 11-20, 2002.

[42] Y. Du, J. Chen, J. L. Funderburgh, X. Zhu, and L. Lingsong, "Functional reconstruction of rabbit corneal epithelium by human limbal cells cultured on amniotic membrane," Molecular Vision, vol. 9, pp. 635-643, 2003.

[43] E. M. Espana, M. A. di Pascuale, H. He et al., "Characterization of corneal pannus removed from patients with total limbal stem cell deficiency," Investigative Ophthalmology and Visual Science, vol. 45, no. 9, pp. 2961-2966, 2004.

[44] D. G. Harkin, Z. Barnard, P. Gillies, S. L. Ainscough, and A. J. G. Apel, "Analysis of p63 and cytokeratin expression in a cultivated limbal autograft used in the treatment of limbal stem cell deficiency," British Journal of Ophthalmology, vol. 88, no. 9, pp. 1154-1158, 2004.

[45] R. A. Thoft and J. Friend, "The X, Y, Z hypothesis of corneal epithelial maintenance," Investigative Ophthalmology and Visual Science, vol. 24, no. 10, pp. 1442-1443, 1983.

[46] G. Cotsarelis, S.-Z. Cheng, G. Dong, T.-T. Sun, and R. M. Lavker, "Existence of slow-cycling limbal epithelial basal cells that can be preferentially stimulated to proliferate: Implications on epithelial stem cells," Cell, vol. 57, no. 2, pp. 201-209, 1989.

[47] Y. Li, T. Inoue, F. Takamatsu et al., "Differences between niche cells and limbal stromal cells in maintenance of corneal limbal stem cells," Investigative Ophthalmology and Visual Science, vol. 55, no. 3, pp. 1453-1462, 2014.

[48] H. J. Levis, R. A. Brown, and J. T. Daniels, "Plastic compressed collagen as a biomimetic substrate for human limbal epithelial cell culture," Biomaterials, vol. 31, no. 30, pp. 7726-7737, 2010.
[49] J. J. Yoon, S. Ismail, and T. Sherwin, "Limbal stem cells: central concepts of corneal epithelial homeostasis," World Journal of Stem Cells, vol. 6, no. 4, pp. 391-403, 2014.

[50] F. Majo, A. Rochat, M. Nicolas, G. A. Jaoudé, and Y. Barrandon, "Oligopotent stem cells are distributed throughout the mammalian ocular surface," Nature, vol. 456, no. 7219, pp. 250-254, 2008.

[51] A. Amitai-Lange, A. Altshuler, J. Bubley, N. Dbayat, B. Tiosano, and R. Shalom-Feuerstein, "Lineage tracing of stem and progenitor cells of the murine corneal epithelium," Stem Cells, vol. 33, no. 1, pp. 230-239, 2015.

[52] S. Kameishi, H. Sugiyama, M. Yamato et al., "Remodeling of epithelial cells and basement membranes in a corneal deficiency model with long-term follow-up," Laboratory Investigation, vol. 95, no. 2, pp. 168-179, 2014.

[53] J. D. West, N. J. Dorà, and J. M. Collinson, "Evaluating alternative stem cell hypotheses for adult corneal epithelial maintenance," World Journal of Stem Cells, vol. 7, no. 2, pp. 281299, 2015.

[54] J. M. Wolosin, "Cell markers and the side population phenotype in ocular surface epithelial stem cell characterization and isolation," Ocular Surface, vol. 4, no. 1, pp. 10-23, 2006.

[55] U. Schlötzer-Schrehardt and F. E. Kruse, "Identification and characterization of limbal stem cells," Experimental Eye Research, vol. 81, no. 3, pp. 247-264, 2005.

[56] B. R. Ksander, P. E. Kolovou, B. J. Wilson et al., “ $A B C B 5$ is a limbal stem cell gene required for corneal development and repair," Nature, 2014.

[57] J. R. Sharpe, S. M. Daya, M. Dimitriadi, R. Martin, and S. E. James, "Survival of cultured allogeneic limbal epithelial cells following corneal repair," Tissue Engineering, vol. 13, no. 1, pp. 123-132, 2007.

[58] O. Baylis, F. Figueiredo, C. Henein, M. Lako, and S. Ahmad, "13 Years of cultured limbal epithelial cell therapy: a review of the outcomes," Journal of Cellular Biochemistry, vol. 112, no. 4, pp. 993-1002, 2011. 

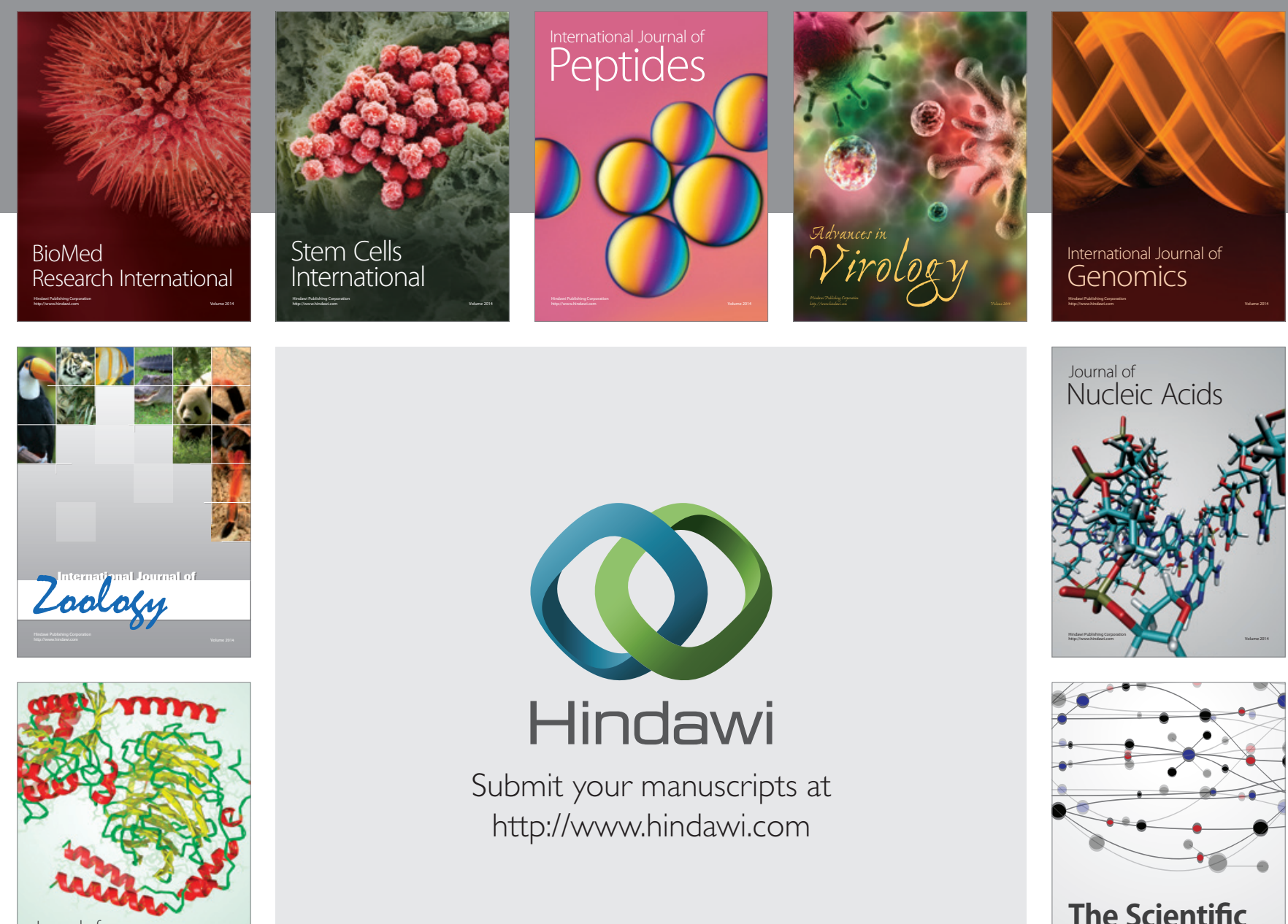

Submit your manuscripts at

http://www.hindawi.com

Journal of
Signal Transduction
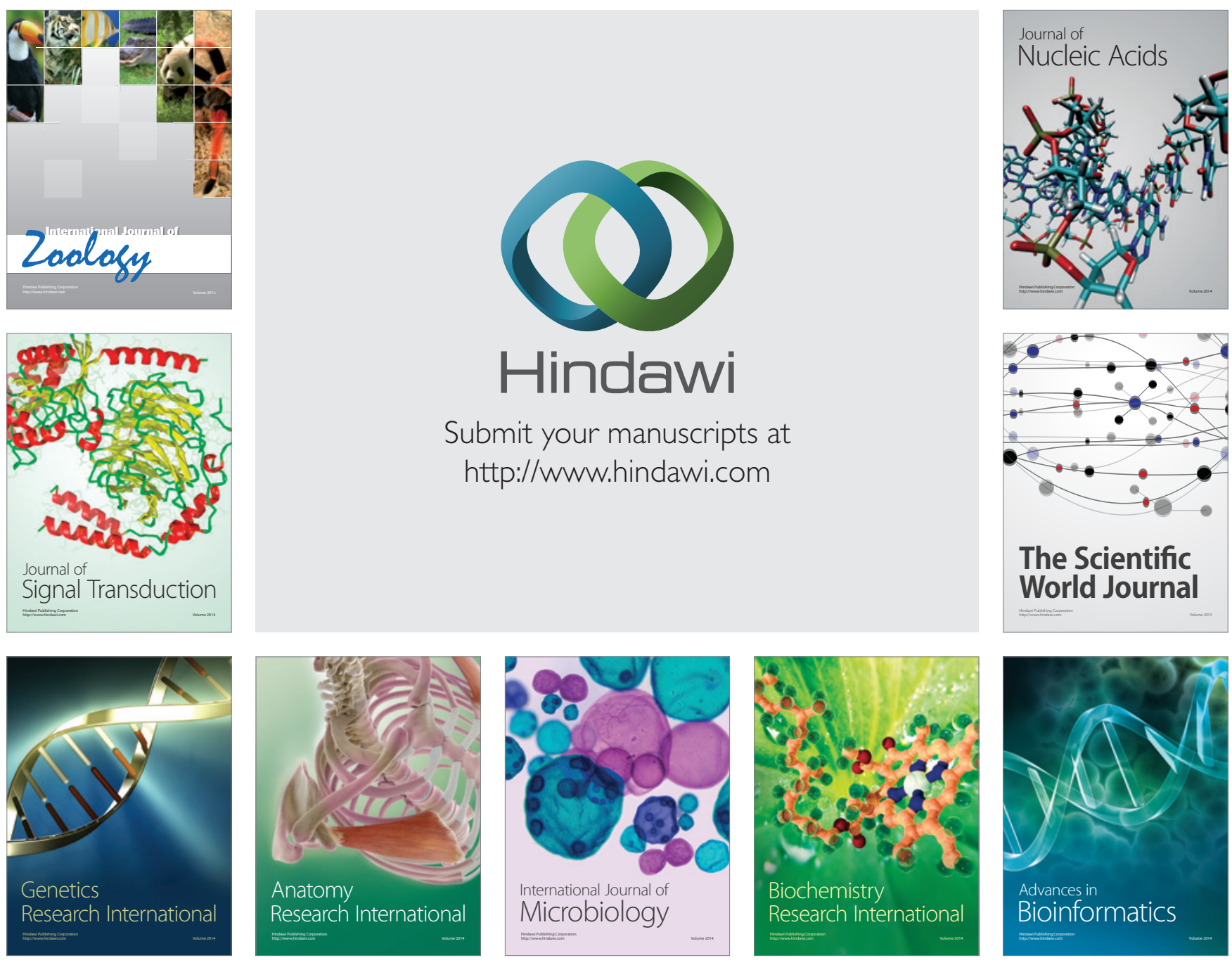

The Scientific World Journal
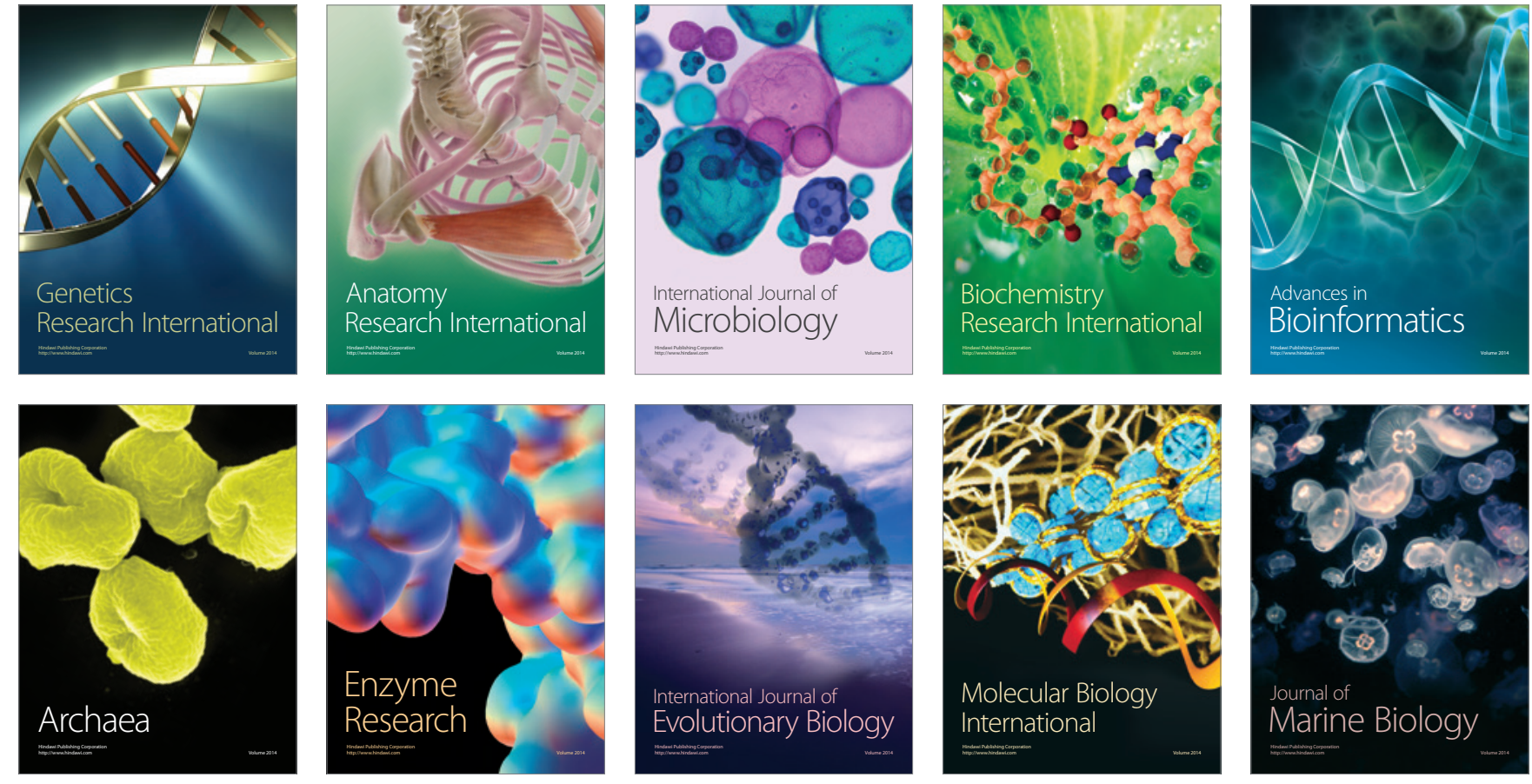